\title{
Gesundheit und Arbeit - Gesundheit am Arbeitsplatz
}

\author{
Anne-Geneviève Bütikofer
}

lic. iur., Generalsekretärin der FMH

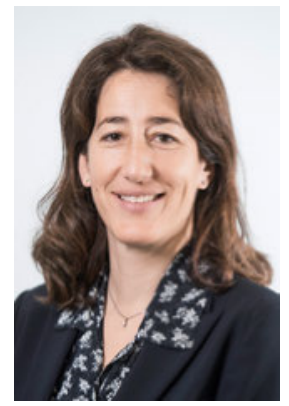

Die Gesundheit am Arbeitsplatz betrifft uns alle - ob als Arbeitgeber, Arbeitnehmer oder Kollegen. Arbeiten ist gut für die Gesundheit, die Arbeitsbedingungen können diese allerdings beeinträchtigen. Die Gesundheit am Arbeitsplatz betrifft uns ebenso als Individuen, Partner oder Elternteile, zumal sie direkt unser Privatleben beeinflusst. Unabhängig vom ausgeübten Beruf oder von der bekleideten Funktion und Position können sich zahlreiche Faktoren auf die körperliche und psychische Integrität der Menschen auswirken und unterschiedlichste Folgen oder psychosoziale Risiken nach sich ziehen: Stress, Erschöpfung, Mobbing bis hin sogar zu Belästigung.

Die rasante Entwicklung der Arbeitswelt sowie der Anstieg der wirtschaftlichen Zwänge in den vergangenen Jahren führen dazu, mit immer weniger Mitteln immer mehr leisten zu müssen. Im Arbeitsumfeld nimmt der Druck stetig zu, was häufig mit seelischen Folgen einhergeht. Die Arbeit im Gesundheitswesen bleibt davon nicht ausgenommen.

Die Internationale Arbeitsorganisation (ILO) und das Staatssekretariat für Wirtschaft (seco) machen auf die Arbeitsbedingungen aufmerksam, insbesondere Überbelastung, unvereinbare Ansprüche und mangelnde Klarheit über die auszuübende Tätigkeit. Hinzu kommen der fehlende Einbezug der Arbeitnehmenden in Entscheidungen, die sie betreffen, und ihr mangelnder Einfluss auf die Umstände, wie sie ihren Beruf ausüben, schlecht durchgeführte Umstrukturierungen und eine unsichere Beschäftigungslage, wirkungslose Kommunikation und mangelnde Unterstützung durch Führung oder Kollegen. Solche Bedingungen können die oben genannten Risiken bewirken.

Unsere Gesellschaft hat sich weiterentwickelt; jeder von uns möchte Privat- und Berufsleben besser miteinander vereinbaren. Das Generalsekretariat der FMH verfolgt diese Fragen mit besonderer Aufmerksamkeit. 2013 hat es ein Projekt zum betrieblichen Gesundheitsmanagement lanciert, mit der Absicht, das Label «Friendly Workspace» der Stiftung für Gesundheitsförderung Schweiz zu erhalten. Ziel ist dabei, einen Arbeitsort sicherzustellen, in dem Arbeitnehmende, Kader und Leitung gemeinsam kontinuierlich darauf hinarbeiten, die Gesundheit, die Sicherheit und das Wohlergehen aller sowie die Nachhaltigkeit des Arbeitsortes zu schützen und zu fördern. Auch wenn es nicht perfekt und noch nicht einmal abgeschlossen ist, hat dieses Projekt doch bereits bewirkt, dass wir der Gesundheit, der Sicherheit und dem Wohlergehen im psychosozialen Arbeitsumfeld deutlich mehr Aufmerksamkeit widmen, auch was die Organisation der Arbeit und die Unternehmenskultur angeht.

Im Laufe der vergangenen Jahre haben darüber hinaus eine Reihe von Beiträgen und Leitartikeln der Abteilung Public Health der SÄZ (2015;96[25]:899/2016;97[5]: 167) in Erinnerung gerufen, dass Ärztinnen und Ärzte in ihrem Arbeitsalltag einer erheblichen psychosozialen Belastung ausgesetzt sind und dass Veränderungen ihrer Arbeitsbedingungen sich häufig in einem Burnout niederschlagen, wo doch ihre Gesundheit von so grosser Bedeutung ist. In dieser Hinsicht wird die FMH unter Führung der Abteilung Public Health ihren Einsatz in diesem Bereich fortsetzen und weiterhin Symposien "Gesunde Ärzte» organisieren, um zur Förderung und Wahrung der ärztlichen Gesundheit beizutragen.

Ob ein junger Arzt, eine erfahrene Ärztin oder ein Mitarbeiter des GS der FMH - sie alle schöpfen Energie daraus und finden Gefallen daran, wenn sie zusammenarbeiten, neue Lösungen finden und letztlich ihre Ziele erreichen. Es ist also nicht nur eine gesetzliche Verpflichtung, sondern ergibt sich aus unserer sozialen Verantwortung gegenüber allen, in der Arbeit mit Kolleginnen, Arbeitnehmenden und Patienten alle notwendigen Massnahmen zu ergreifen, um die persönliche Integrität zu schützen und psychosoziale Risiken zu mindern. Dadurch werden die Freude am Arbeiten und das Vertrauen gegenüber den Arbeitskollegen erhalten, das Leistungswachstum gefördert und die beruflichen Begabungen und Chancen genutzt - kurz: alle verhängnisvollen Auswirkungen auf das Berufs- und Privatleben jeder und jedes Einzelnen werden vermieden.

Ich hoffe, dass diese Überlegungen in unsere guten Vorsätze zum Jahreswechsel einfliessen, und wünsche allen Leserinnen und Lesern schöne und erholsame Festtage und einen guten Start ins neue Jahr. 